\title{
Myopia prevalence in Canadian school children: a pilot study
}

\author{
Mike Yang $\mathbb{D}^{1} \cdot$ Doerte Luensmann $^{1} \cdot$ Desmond Fonn $^{1} \cdot$ Jill Woods $^{1} \cdot$ Debbie Jones $^{1} \cdot$ Keith Gordon $^{2} \cdot$ Lyndon Jones $^{1}$
}

Received: 23 December 2016 / Accepted: 17 December 2017 / Published online: 2 February 2018

(c) The Royal College of Ophthalmologists 2018

\begin{abstract}
Purpose A pilot study to determine the prevalence of myopia, proportion of uncorrected myopia and pertinent environmental factors among children in a suburban region in Canada.

Methods Refraction with cycloplegia and ocular biometry were measured in children of two age groups. Myopia was considered at a spherical equivalent refraction $(\mathrm{SER}) \leq-0.50 \mathrm{D}$ in at least one eye. Parents completed a questionnaire that captured the child's daily activities.

Results A total of 166 children completed the study (83 aged 6-8 and 83 aged 11-13). Myopia prevalence was 17.5\% among the overall group, $6.0 \%$ among ages 6-8 and $28.9 \%$ among ages 11-13. Mean subjective SER in myopic children was $-1.10 \mathrm{D}(95 \%$ confidence interval $(\mathrm{CI}),-0.34$ to $-1.86 \mathrm{D})$ at ages $6-8$ and $-2.44 \mathrm{D}(95 \% \mathrm{CI},-1.71$ to $-3.18 \mathrm{D})$ at ages 11-13. In this study, 34.5\% of the myopic children were uncorrected, which represented $6.0 \%$ of the entire group of children. Mean axial length (AL) increased by $1.03 \mathrm{~mm}$ from ages 6-8 (mean $22.62 \mathrm{~mm}$; $95 \% \mathrm{CI}, 22.45$ to $22.79 \mathrm{~mm}$ ) to ages 11-13 (mean $23.65 \mathrm{~mm} ; 95 \% \mathrm{CI}, 23.45$ to $23.84 \mathrm{~mm} ; p<0.01$ ). The correlation coefficient between AL and SER was $-0.618(p<0.01)$. Binary logistic regression between outdoor time and the prevalence of myopia showed that one additional hour of outdoor time per week lowered the odds of a child having myopia by $14.3 \%$ $(p=0.007)$.
\end{abstract}

Conclusion Myopia prevalence increased from 6\% at ages 6-8 to $29 \%$ at ages 11-13. Thirty-five per cent of the myopes in this study were uncorrected. More time outdoors may be beneficial to protect against myopia onset.

\section{Introduction}

Myopia or nearsightedness is a common refractive error seen worldwide and has increased over the last few decades $[1,2]$. Prevalence of myopia among adults in the United States increased by nearly 17\% from 1972 to 2004, and similar increases were found in Taiwan over a time span of only 20 years $[1,3]$. The prevalence of myopia in 5-17 year olds in the USA is $\sim 9 \%$, ranging from 4 to $19 \%$ depending on the ethnic background [4], and is almost $50 \%$ for 20-39 year olds [5]. Prevalence of myopia is $14 \%$ in Ireland and $13 \%$ in Australia among 11-17 year olds [2, 6]. In the same age group, East Asia has significantly higher rates of myopia, reaching 49\% in China [7], 63\% in Hong Kong [8]

Mike Yang

mike.yang@uwaterloo.ca

1 Centre for Ocular Research \& Education, School of Optometry \& Vision Science, University of Waterloo, Waterloo, ON, Canada

2 Canadian National Institute for the Blind, Toronto, ON, Canada and a prevalence up to $95 \%$ among university students in China [9].

There is still no conclusive evidence to define the roles of genetic and environmental factors on myopia development. Strong associations between parental myopia and myopia development in children have been published [10, 11], and factors such as time spent studying, watching TV, using computers and physical activities outdoors have been reported to have possible environmental influences on myopia [12]. Conversely, studies from China and Australia found a weak or absent association between prolonged near work and myopia progression [6, 13]. Besides attempting to control these modifiable environmental risk factors, much of the current myopia control research focuses on slowing the progression of myopia by halting ocular axial length growth, using optical or pharmaceutical methods [14, 15].

Increased incidence of myopia comes with a heavy economic burden. The annual cost of vision loss in Canada was estimated to be $\$ 15.8$ billion in 2007 [16], with refractive error accounting for $>60 \%$ of health system expenditures. The increased number of high myopes with a refractive error of more than $-5.00 \mathrm{D}$ resulted in an increase 
in related pathological complications such as myopic retinopathy, retinal detachment and glaucoma [17]. In the Rotterdam eye study, myopia was identified as the most important cause of impaired vision in persons under the age of 75 [18]. Of further concern is that the onset of myopia has shifted to a younger age [2, 3]. Among children aged 6-7 years old, the prevalence was $2 \%$ in Ireland and Finland, and $6 \%$ in China $[2,7,19]$. The early onset of myopia means these young eyes will spend more time during the rapid progressive phase of myopia development in childhood, leading to possibly even higher degrees of myopia than currently observed, and potentially more visiondebilitating diseases related to high myopia in later life.

The population of Canada is growing by nearly 3 million people every 10 years, primarily due to immigration [20]. More than two-thirds of the immigrants originate from countries with a high prevalence of myopia, including East Asian countries and Europe [21]. Previous prevalence research in Canada was limited to retrospective chart reviews, studies involving only clinic patients and some without cycloplegic accommodative control [22-24]. This study is the first population-based study designed to examine myopia prevalence in Canadian school-aged children.

\section{Methods}

\section{Participant recruitment}

This study was a cross-sectional myopia prevalence study targeting two age groups. The age groups targeted for enrolment were school children in grades 1 and 2 (age 6-8), and grades 6 and 7 (age 11-13). In all, 165 elementary schools in the Waterloo Region were randomly selected and contacted for the study, out of which 10 gave permission to carry out the study at the school. All children in grades 1-2 and 6-7 during the 2014-2015 academic year were invited to participate, and the combined population in those two age groups was 1526 . The 10 schools that agreed to participate were geographically spread out throughout the sub-areas of the Waterloo Region, and there is no distinct difference between populations in these schools and the rest of the 165 schools based on available public records. Parents provided written informed consent and the child provided assent prior to any study procedures being undertaken. Children were screened at the schools and the exclusion criteria used were as follows: previous refractive surgery or orthokeratology; known sensitivity to diagnostic pharmaceutical drops used in the study; serious medical disorders; and abnormalities such as amblyopia and cataract.

Approval of this project was granted through the Office of Research Ethics at the University of Waterloo, and through the research committees of the local district school boards. Each school principal also approved the study prior to participant recruitment. All procedures adhered to the Declaration of Helsinki.

\section{Definition of myopia}

Spherical equivalent refraction (SER) was used to report refractions in this study, which is the sum of the spherical value plus half the cylindrical value. Myopia was defined as having a cycloplegic subjective SER more minus than or equal to $-0.50 \mathrm{D}$ in at least one eye. This definition is the same as that used in previous paediatric myopia prevalence studies [25, 26].

\section{Measurements}

This study included up to two study visits. The first visit was completed at the schools, at which testing was undertaken without cycloplegia. The participants who had a subjective SER $\leq-0.50 \mathrm{D}$ in at least one eye at the first visit were invited to a second study visit at the Centre for Ocular Research \& Education, where their refraction was repeated under cycloplegia. The aim of the second visit was to measure the cycloplegic refractive error and identify any false positives who were myopic in the first visit due to accommodative spasm. The following measurements were captured at the first visit: automated refraction using RetinoMax K-Plus handheld autorefractor (Nikon Corp. Tokyo, Japan); subjective refraction using trial lens set (B\&H Optical, Belleville, Canada); visual acuity using ETDRS chart (Precision Vision, IL, USA); and ocular biometry using IOLMaster (Carl Zeiss Meditec, Jena, Germany). At least eight measurements per eye were made with the autorefractor, and an average of the measurements was automatically calculated. Acuity was measured at $4 \mathrm{~m}$, which is the manufacturer-specified distance for the chart used. Minus power was only increased during subjective refraction if the participants' acuity improved by three or more letters. The IOLMaster uses partial coherence interferometry to measure the axial length of the eye, and dual mode was selected to include keratometry ( $K$; aka: central corneal curvature). Average $K$ was calculated by adding half of the difference between flat and steep $K$ to the flat $K$ value. The autorefractor and IOLMaster were both calibrated using their respective model eye on each day of measurement.

At the second study visit, cycloplegia was achieved using a protocol described and validated by Egashira et al. [27]. The eyes were first anesthetised using one drop of $0.5 \%$ proparacaine hydrochloride (Alcaine, Alcon) instilled in both eyes. After $1 \mathrm{~min}$, one drop of 1\% Tropicamide Ophthalmic Solution USP (Mydriacyl, Alcon) was instilled. 
After 5 min another drop of $1 \%$ Tropicamide was instilled. After waiting $25 \mathrm{~min}$ following the last drop instillation, autorefraction was measured three times per eye using a table-mounted KR8800 auto-refractometer (Topcon Corp. Tokyo, Japan), and an average of the three measurements was automatically calculated. Subjective refraction and ocular biometry were then repeated with a phoropter and IOLMaster.

\section{Questionnaire}

Two to three weeks before the initial tests at the schools, child activity questionnaires were distributed in class. Parents completed these questionnaires and returned them to the research centre before testing took place. Parents were asked about specific child activities that may be an environmental influence on myopia development. Questions included the number of hours the child spent per week on reading, watching $\mathrm{TV}$, using a computer, indoor sport activities and outdoor activities. Parents were asked to identify whether one or both parents were myopic. At the start of testing at the schools, children were asked whether they currently wear glasses or contact lenses to correct refractive error.

\section{Statistics}

A double data entry system was used and any inconsistencies or data outside the expected range were checked against source documents. Data analysis was conducted using Statistica 13 (Dell Inc., USA) and SPSS (IBM, Inc., USA). Descriptive statistics (mean and SD) were conducted for age. Mean and 95\% confidence interval (CI) were reported for refractions, corneal curvature and axial length in the more myopic eye in each age group. A twosample independent $t$-test was used to compare refraction and axial length of the two age groups recruited in the study.

Environmental factors that may affect myopia development such as reported time spent on school work, reading, watching TV, computer use, outdoor and indoor sports were analysed using simple binary logistic regression. These factors were also analysed for their correlation with SER. The percentage change in odds of having myopia was calculated by subtracting $\operatorname{Exp}(B)$ from the value 1.00 (the exponent beta coefficient is taken from the SPSS output of the linear regression). The difference represented the percentage change in odds of having myopia per 1 unit increase in one of the environmental factors. Correlation between axial length and SER was determined using Pearson product-moment correlation in Statistica. All statistical tests were two-sided, and a $p$-value of $\leq 0.05$ was considered significant.

\section{Results}

In total, 174 children were enroled, of which 1 was excluded due to a previous history of amblyopia. Seven children were later discontinued from the study due to apprehension about eye drops or they were lost to follow-up. Data from 166 participants who completed the study were analysed. Equal numbers of children from the two targeted grade groups participated (83 participants from grade 1-2 and 83 participants from grade 6-7). There were also similar numbers of males and females in the study ( 82 males and 84 females). The study population was $80.1 \%$ Caucasian, $6.6 \%$ East Asian, 4.8\% South Asian, 0.6\% African American and $7.1 \%$ with mixed ethnic background or other not specified background. The mean \pm SD ages were $9.6 \pm 2.5$ years for the overall study population, $7.2 \pm 0.6$ for grade $1-2$ (ages 6-8 group) and $12.1 \pm 0.6$ for grade 6-7 (ages 11-13 group).

The overall prevalence of myopia in the study population was $17.5 \%$ (95\% CI, 11.7-23.2\%). The prevalence of myopia in children aged 6-8 was $6.0 \%(95 \%$ CI, $0.9-11.1 \%)$ and in children aged $11-13$ was $28.9 \%(95 \%$ CI, 19.2-38.7\%). Prevalence in male students was $4.7 \%$ in ages $6-8$ and $28.2 \%$ in ages 11-13. Prevalence in female students was $7.5 \%$ in ages $6-8$ and $29.5 \%$ in ages $11-13$. Out of the 29 participants who were myopic in the study, 10 were uncorrected prior to the study, which amounted to $34.5 \%$ of the myopic children or $6.0 \%$ of the overall group.

In the older children, in addition to a higher prevalence of myopia, the degree of myopia was also greater. When data from the more myopic eye (or the least hyperopic eye) of all children were examined, mean subjective SER for 6-8 year olds was $+0.18 \mathrm{D}(95 \% \mathrm{CI}, 0.00$ to $+0.36 \mathrm{D})$ and mean SER for 11-13 year olds was $-0.58 \mathrm{D}(95 \% \mathrm{CI}$, -0.93 to $-0.22 \mathrm{D})$. This difference was statistically significant $(p<0.001)$.

For children who met the definition of myopia, the mean subjective SER was $-1.10 \mathrm{D}(95 \% \mathrm{CI},-1.86$ to $-0.34 \mathrm{D})$ in children aged $6-8$, and $-2.44 \mathrm{D}(95 \% \mathrm{CI},-3.18$ to $-1.71 \mathrm{D})$ in children aged $11-13$. The difference of -1.34 $\mathrm{D}$ between the two groups was not statistically significant ( $p=0.104)$, however the statistical insignificance may have been a type 2 statistical error due to the small sample size.

Six child activities were analysed for their effect on the odds of myopia. Outdoor time was the only statistically significant factor, with one additional hour of outdoor time per week lowering the odds of myopia by $14.3 \%$ (odds ratio (OR), 0.857; 95\% CI, 0.766-0.959; $p=0.007$ ). School work, reading for pleasure, watching TV and using a computer correlated negatively with SER, but were not statistically significant. Indoor sport activities correlated positively with SER but this was also not statistically significant. Aside from these environmental factors, parental 
myopia had a strong influence on the child's refractive error. Children were 2.52 times more likely to be myopic if at least one parent had myopia, compared to children with two non-myopic parents $(\mathrm{OR}=2.524 ; 95 \% \mathrm{CI}, 1.078-5.912, p$ $=0.033$ ).

Overall, the mean axial length was $22.62 \mathrm{~mm}$ (95\% CI, $22.45-22.79 \mathrm{~mm}$ ) in children aged $6-8$, and $23.65 \mathrm{~mm}$ (95\% CI, 23.45-23.84 mm) in children aged 11-13. The difference was statistically significant, $p<0.001$. Among the myopic children, mean axial length was $22.95 \mathrm{~mm}(95 \%$ CI, 22.39-23.50 mm) for the 6-8 year olds, and $24.29 \mathrm{~mm}$ (95\% CI, 23.97-24.61 mm) for the 11-13 year olds. The difference was also statistically significant, $p<0.001$. Axial length correlated negatively with SER $(r=-0.618, p<$ 0.001 ), meaning with a longer axial length, SER became more negative (more myopic).

Central corneal curvature or keratometry $(K)$ for females was steeper for both age groups. For ages $6-8$, average $K$ for males was $43.27 \mathrm{D}(95 \% \mathrm{CI}, 42.86-43.68 \mathrm{D})$ and for females was $44.09 \mathrm{D}$ (95\% CI, 43.55-44.63 D), with female corneas being steeper by $0.82 \mathrm{D}(p=0.016)$. For ages $11-13$, average $K$ for males was $42.78 \mathrm{D}$ (95\% CI, 42.39-43.17 D) and average $K$ for females was $43.71 \mathrm{D}$ (95\% CI, 43.33-44.10 D), with female corneas being steeper by $0.93 \mathrm{D}(p<0.001)$. Despite the difference between males and females, there was no statistically significant difference between the two age groups: average $K$ for ages 6-8 was 43.67 D (95\% CI, 43.33-44.01) and average $K$ for ages $11-13$ was $43.27 \mathrm{D}(95 \% \mathrm{CI}, 42.99-43.56 \mathrm{D})$, with a difference of $-0.40 \mathrm{D}(p=0.077)$.

\section{Discussion}

Fewer than 14\% of children in Canada under age 6 have had eye examinations by an eye care practitioner [28], yet according to our study findings, more than one in six children between age 6 and 13 are myopic. Comparing these pilot data to other studies with similar definition for myopia and age group, we found a higher prevalence of myopia $(17.5 \%)$ than reported in the USA $(9.2 \%)$ [4], but less than that reported in Korea (46.5\%) [29]. Generally, myopia in children is more prevalent in East Asians, with 38.1\% reported in southern China [7], 63.3\% in Hong Kong [8] and $30 \%$ in Taiwan [30]. The high prevalence in Eastern Asia can be contrasted with 9.9\% in Finland [19], 12.8\% in Australia [6] and $14.6 \%$ in Ireland [2].

In this study, a child was categorised as having uncorrected myopia if he/she was found to have myopia $(\leq-0.50 \mathrm{D}$ SER) in at least one eye but did not have spectacles or any other refractive correction prior to enrolment into the study. Using this definition, $34.5 \%$ of the children deemed myopic in this cohort were found to be uncorrected, which represented $6.0 \%$ of the overall group of children tested.

In our study, myopia prevalence increased significantly with age from $6.0 \%$ in children aged $6-8$ to $28.9 \%$ in children aged 11-13. Other studies found myopia further increased in prevalence from age 14 and onwards: in Ireland prevalence increased from $1.9 \%$ in ages $6-7$ to $14.6 \%$ in ages $12-13$ and later to $18.6 \%$ in ages $18-20$ [2]. The global prevalence of myopia is estimated to have increased from $14 \%$ in ages $10-14$ to $37 \%$ in ages $20-24$ [31], and the prevalence is $47.2 \%$ in Europe for those aged 25-29 [32]. In this study, although the increase in SER from the younger age group to the older for the total sample was significant, the increase in myopia among myopic children by $-1.34 \mathrm{D}$ from the 6-8 year olds to the 11-13 year olds was not significant. We propose that the disparity between these results is because of the considerably smaller sample of myopic children and relatively large and overlapping confidence intervals, suggesting that the increase in myopia will be significant with a larger sample. This is demonstrated in the total sample.

A previous Canadian study reviewed paediatric patient charts from 2007 at a teaching clinic, and found myopia prevalence to be $13.6 \%$ in $5-10$ year olds and $42.2 \%$ in 10-15 year olds [23]. Another Canadian study reviewed patient charts (only Chinese-Canadian children) from 2003 at a private practice in Mississauga, $\mathrm{ON}$, and found myopia prevalence increased from $22.4 \%$ at age 6 to $64.1 \%$ at age 12 [24]. These two studies were on clinical populations presenting for examination and thus the prevalence would be expected to be inflated compared to this study, which sampled participants from the general population of schoolaged children. However, the sample size in our study was small compared to other prevalence studies, and this limited our ability to narrow the $\mathrm{CI}$ of the results. For future studies, a larger sample size would require new public health policies to allow vision testing of every child in a particular grade in schools.

Time spent outdoors was the only surveyed child activity that correlated with refraction by regression analysis, exhibiting a weak statistically significant correlation $(r=$ $0.264, p=0.001)$. There were no significant associations between indoor sports and myopia, so it was time spent outdoors rather than actually playing sports, which appeared to have an impact on myopia development. In this study, every additional hour spent outdoors per week was found to lower the odds of myopia development by $14.3 \%$. Studies in Australia, China and Ireland reported similar results, where more outdoor activities were found to reduce the prevalence of myopia [6, 33, 34].

When at least one parent was myopic, the child was 2.52 times more likely to develop myopia compared to a child with two non-myopic parents. This predictive value 
indicates that genetics continue to play an important role in the development of myopia. These results indicate that it is a combination of both genetics and the child's environment that determines the final visual outcome [29, 33, 35].

Axial length was negatively associated with SER $(r=$ $-0.618, p<0.01$ ), demonstrating that longer axial length was associated with higher myopia. Females had shorter axial length compared to males $(\Delta=-0.36 \mathrm{~mm}, p=$ $0.019)$, but there was a trend of more myopic SER in females $(\Delta=-0.23 \mathrm{D}, p=0.275)$. This discrepancy can be partially explained by females having a steeper corneal curvature $(\Delta=0.86 \mathrm{D}, p<0.001)$. Axial length increased from the younger group of children to the older group $(\Delta=$ $1.03 \mathrm{~mm}, p<0.01$ ), therefore slowing the increase in axial length appears to be an obvious target for therapy to slow the development of myopia. The exact mechanism behind abnormal myopia-inducing axial length growth is still unknown and remains an active research area $[14,15]$.

Ongoing prevalence studies in key Canadian locations are recommended, as Canada has a unique and dynamic demographic that is different from the neighbouring USA, which has a higher percentage of African American, Hispanic and Latino populations [36]. The demographics of the population in this study (Waterloo Region) was comparable to that of Canada from a recent census [37]. For example, while the study population contained $81.0 \%$ Caucasians and $6.6 \%$ East Asians, the reported Canadian population contained $80.9 \%$ Caucasians and 5.7\% East Asians. Larger national studies should include children living in urban and rural areas, and cover different provinces for a better representation of myopia prevalence in school children across Canada.

Understanding the current prevalence of myopia allows us to raise awareness and educate communities at local, provincial and/or federal levels. This knowledge may further be used to estimate the financial burden for vision correction and pathological complications associated with high myopia. While there are no currently approved methods to reduce existing myopia, numerous studies are currently investigating options to slow the progression of myopia using various pharmaceutical and optical treatment methods $[14,15,38]$.

\section{Conclusion}

This pilot study estimated myopia prevalence to be $6.0 \%$ in ages 6 through 8 , increasing to $28.9 \%$ in ages 11 through 13 in the Waterloo Region. In total, $6.0 \%$ of the children tested had uncorrected myopia. Time spent outdoors was the only child activity to have a significant impact on myopia, and one additional hour of outdoor time per week lowered the odds of having myopia by $14.3 \%$ in the age groups investigated in this study. When at least one parent was myopic, the child was 2.52 times more likely to develop myopia compared to a child with two non-myopic parents. Axial length increased as myopia increased and is therefore a good target for intervention and control of myopia development. These study results have many potential implications for academic researchers, educators and healthcare policy makers, and to our knowledge, this pilot study is the first of its kind studying the general population of school-aged children in Canada. Larger national studies would be able to extract more detailed information on various age and ethnic groups and provide evidence based recommendations to the general public and healthcare stakeholders.

\section{Summary}

\section{What was known before}

Myopic prevalence increased in the USA and Europe over the past few decades and higher prevalence of myopia is reported in Asia.

Environmental and genetic factors may be associated with myopia development.

High myopia comes with heavy economic burden.

\section{What this study adds}

Myopia prevalence was $6.0 \%$ in ages 6 through 8 , increasing to $28.9 \%$ in ages 11 through 13 in the Waterloo Region in Canada.

Six per cent of the children tested had uncorrected myopia.

More time spent outdoors lowered the odds of having myopia. Child with myopic parents were more likely to develop myopia. Axial length increased as myopia increased.

Acknowledgements We thank the two School Boards in the Waterloo Region and their participating schools for their contributions to this study, and Carl Zeiss Canada for providing an IOLMaster on loan. We are grateful to Dominik Papinski for his help with statistical analysis.

Funding This study was partially funded by a grant from Essilor and by the Centre for Ocular Research \& Education, University of Waterloo.

\section{Compliance with ethical standards}

Conflict of interest The authors declare that they have no competing interests.

\section{References}

1. Vitale S, Sperduto RD, Ferris FLI. Increased prevalence of myopia in the United States between 1971-1972 and 1999-2004. Arch Ophthalmol. 2009;127:1632-9. 
2. McCullough SJ, O’Donoghue L, Saunders KJ. Six year refractive change among white children and young adults: evidence for significant increase in myopia among white UK children. PLoS ONE. 2016;11:e0146332.

3. Lin LL, Shih YF, Hsiao CK, Chen CJ. Prevalence of myopia in Taiwanese schoolchildren: 1983 to 2000. Ann Acad Med Singapore. 2004;33:27-33.

4. Kleinstein RN, Jones LA, Hullett S, et al. Refractive error and ethnicity in children. Arch Ophthalmol. 2003;121:1141-7.

5. Vitale S, Ellwein L, Cotch MF, Ferris FLI, Sperduto R. Prevalence of refractive error in the United States, 1999-2004. Arch Ophthalmol. 2008;126:1111-9.

6. Rose KA, Morgan IG, Ip J, et al. Outdoor activity reduces the prevalence of myopia in children. Ophthalmology. 2008;115:1279-85.

7. He M, Zeng J, Liu Y, Xu J, Pokharel GP, Ellwein LB. Refractive error and visual impairment in urban children in southern China. Invest Ophthalmol Vis Sci. 2004;45:793-9.

8. Lam CSY, Edwards M, Millodot M, Goh WSH. A 2-year longitudinal study of myopia progression and optical component changes among Hong Kong schoolchildren. Optom Vis Sci. 1999;76:370-80.

9. Sun J, Zhou J, Zhao P, et al. High prevalence of myopia and high myopia in 5060 Chinese university students in Shanghai. Invest Ophthalmol Vis Sci. 2012;53:7504-9.

10. Lim LT, Gong Y, Ah-Kee EY, Xiao G, Zhang X, Yu S. Impact of parental history of myopia on the development of myopia in mainland China school-aged children. Ophthalmol Eye Dis. 2014;6:31-35.

11. Mutti DO, Mitchell GL, Moeschberger ML, Jones LA, Zadnik K. Parental myopia, near work, school achievement, and children's refractive error. Invest Ophthalmol Vis Sci. 2002;43:3633-40.

12. French AN, Morgan IG, Mitchell P, Rose KA. Risk factors for incident myopia in Australian schoolchildren: the Sydney adolescent vascular and eye study. Ophthalmology. 2013;120:2100-8.

13. Saw SM, Chua WH, Hong CY, et al. Nearwork in early-onset myopia. Invest Ophthalmol Vis Sci. 2002;43:332-9.

14. Smith EL 3rd. Optical treatment strategies to slow myopia progression: effects of the visual extent of the optical treatment zone. Exp Eye Res. 2013;114:77-88.

15. Walline JJ. Myopia control: a review. Eye Contact Lens. 2016;42:3-8.

16. Cruess AF, Gordon KD, Bellan L, Mitchell S, Pezzullo ML. The cost of vision loss in Canada. 2. Results. Can J Ophthalmol. 2011;46:315-8

17. Jones $\mathrm{D}$, Luensmann $\mathrm{D}$. The prevalence and impact of high myopia. Eye Contact Lens. 2012;38:188-96.

18. Klaver CC, Wolfs RC, Vingerling JR, Hofman A, de Jong PT. Age-specific prevalence and causes of blindness and visual impairment in an older population: the Rotterdam Study. Arch Ophthalmol. 1998;116:653-8.

19. Laatikainen L, Erkkilä $H$. Refractive errors and other ocular findings in school children. Acta Ophthalmol (Copenh). 1980;58:129-36.

20. Statistics Canada. Population growths in Canada: From 1851 to $2061 \mathrm{http}: / / \mathrm{www} 12$. statcan.gc.ca/census-recensement/2011/as-sa/ 98-310-x/98-310-x2011003_1-eng.cfm. Accessed 05 July 2012.
21. Statistics Canada. Immigrant population by place of birth and period of immigration (2006 Census) http://www.statcan.gc.ca/ta bles-tableaux/sum-som/101/cst01/demo24a-eng.htm. Accessed 05 July 2012.

22. Robinson BE. Factors associated with the prevalence of myopia in 6-year-olds. Optom Vis Sci. 1999;76:266-71.

23. Hrynchak PK, Mittelstaedt A, Machan CM, Bunn C, Irving EL. Increase in myopia prevalence in clinic-based populations across a century. Optom Vis Sci. 2013;90:1331-41.

24. Cheng D, Schmid KL, Woo GC. Myopia prevalence in ChineseCanadian children in an optometric practice. Optom Vis Sci. 2007;84:21-32.

25. Guo L, Yang J, Mai J, et al. Prevalence and associated factors of myopia among primary and middle school-aged students: a school-based study in Guangzhou. Eye. 2016;30:796-804.

26. Garcia-Lievanos O, Sanchez-Gonzalez L, Espinosa-Cruz N, Hernandez-Flores L, Salmeron-Leal L, Torres-Rodriguez H. Myopia in schoolchildren in a rural community in the State of Mexico, Mexico. Clin Optom. 2016;8:53-56.

27. Egashira SM, Kish LL, Twelker JD, Mutti DO, Zadnik K, Adams AJ. Comparison of cyclopentolate versus tropicamide cycloplegia in children. Optom Vis Sci. 1993;70:1019-26.

28. Breslin DC. Vision loss in Canada 2011. http://www.cos-sco.ca/ wp-content/uploads/2012/09/VisionLossinCanada_e.pdf. National Coalition for Vision Health; 2011. Accessed May 242016.

29. Jang JU. The status of refractive errors in elementary school children in south Jeolla Province, South Korea. Clin Optom. 2015;2015:45-51.

30. Lin LLJ, Hung PT, Ko LS, Hou PK. Study of myopia among aboriginal school children in Taiwan. Acta Ophthalmol (Copenh). 1988;66:34-36.

31. Holden BA, Fricke TR, Wilson DA, et al. Global Prevalence of myopia and high myopia and temporal trends from 2000 through 2050. Ophthalmology. 2016;123:1036-42.

32. Williams KM, Bertelsen G, Cumberland P, et al. Increasing prevalence of myopia in Europe and the impact of education. Ophthalmology. 2015;122:1489-97.

33. O’Donoghue L, Kapetanankis VV, McClelland JF, et al. Risk factors for childhood myopia: findings from the NICER study. Invest Ophthalmol Vis Sci. 2015;56:1524-30.

34. Jin JX, Hua WJ, Jiang X, et al. Effect of outdoor activity on myopia onset and progression in school-aged children in northeast China: the Sujiatun Eye Care Study. BMC Ophthalmol. 2015;15:73.

35. Wojciechowski R. Nature and nurture: the complex genetics of myopia and refractive error. Clin Genet. 2011;79:301-20.

36. Bureau USC. United States Quick Facts. https://www.census.gov/ quickfacts/table/PST045215/00. Accessed 18 May 2016.

37. Statistics Canada. Canada (Code 01) (table). National Household Survey (NHS) Profile. 2011 National Household Survey. http:// www12.statcan.gc.ca/nhs-enm/2011/dp-pd/prof/index.cfm?La ng=E. Statistics Canada Catalogue no 99-004-XWE. Accessed 10 May 2016.

38. Sankaridurg $\mathrm{P}$, Donovan L, Varnas $\mathrm{S}$, et al. Spectacle lenses designed to reduce progression of myopia: 12-month results. Optom Vis Sci. 2010;87:631-41. 